\title{
Short door-in-door-out time of stroke patients in need of thrombectomy in a primary stroke centre
}

\author{
Markku Grönroos ${ }^{1, \star}$, Ville Hällberg ${ }^{1}$, Tuukka Tomminen ${ }^{1}$, Ari Palomäki ${ }^{1,2}$
}

${ }^{1}$ Kanta-Häme Central Hospital,

Department of Emergency Medicine, Ahvenistontie 20, FI-13530

Hämeenlinna, Finland

${ }^{2}$ Tampere University, Faculty of

Medicine and Health Technology,

FI-33014 Tampere University, Finland

\section{*Correspondence}

markku.gronroos@khshp.fi

(Markku Grönroos)

\begin{abstract}
COVID-19 epidemic has had a negative effect on the resources of emergency departments. While the number of acute ischaemic stroke (AIS) patients eligible for reperfusion therapies has decreased in the emergency departments (EDs), times to thrombolytic and endovascular treatments of AIS patients have become longer. In this paper, we report an ultra-fast diagnostic protocol in the settings of AIS in the ED of a primary stroke centre. Experiences using case studies of three patients have also been provided. Patients treated in accordance with the protocol received primary diagnoses and endovascular treatment with door-in-door-out times of 15 to 21 minutes.
\end{abstract}

\section{Keywords}

Acute ischemic stroke; Tissue plasminogen activator; Endovascular treatment; Door-indoor-out-time

\section{Background}

Thrombolysis with tissue plasminogen activator (tPA) should be offered without delay to a suitable patient with acute ischaemic stroke (AIS) [1, 2]. However, in patients with AIS due to large vessel occlusion (LVO), clinical benefit of endovascular treatment (EVT) has been confirmed [3, 4]. Hence, these patients need a very rapid transfer to a centre providing endovascular therapy [5].

Emergency medicine was recognized as an independent speciality in Finland in 2013 [6]. When initiating practical training in this speciality in our emergency department (ED), we implemented a new treatment protocol for acute ischaemic stroke [7]. The protocol is activated on pre-notification by the emergency medical services (EMS), although some AIS patients may arrive by other means. Single call activation prepares the ED, laboratory and computed tomography (CT) staff for AIS patients' reception. The attending physician checks the patient's medical history, notably for contraindications to tPA or thrombectomy. The patient is examined right at the very entrance to the ED, including point-of care INR (POC INR) testing. The patient is then taken to the $\mathrm{CT}$ room where baseline non-contrast computed tomography (NCCT) is performed. In the absence of contraindications, tPA bolus is given in the CT room and computed tomography angiography (CTA) is performed. If large vessel occlusion (LVO) is verified, the attending physician contacts the tertiary hospital. When a decision on potential endovascular treatment has been made, the patient will be transferred by the same EMS unit. The role of emergency physicians in this is decisive, and during the first year of implementation we achieved a median door-to-needle time (DNT) of 20 minutes with good clinical outcomes [8]. Our ED serves as a primary stroke centre (PSC) in a secondary care hospital with no on-site mechanical thrombectomy service. The tertiary hospital with thrombectomy facilities is situated one hour's drive away.

Coronavirus disease 2019 (COVID-19) is caused by the severe acute respiratory syndrome coronavirus 2 (SARS-CoV2). During the first months of 2020, the COVID-19 outbreak occurred in Europe [9], overloaded hospitals and seriously burdened the resources of EDs [10]. SARS-CoV-2 is a respiratory virus which may affect the central nervous system although the mechanism remains unclear. The range of neurological manifestations in patients with COVID-19 varies but these are more common in hospitalized patients [11]. The most common complications are myalgias, headache and encephalopathy along with anosmia and ageusia. Acute cerebrovascular diseases, such as stroke, are emerging as a severe complication. Cohort studies are reporting stroke in 2$6 \%$ of patients hospitalized with COVID-19 [12].

During the COVID-19 epidemic, a decrease was reported in the number of AIS patients eligible for reperfusion therapies, accompanied by increasing delays in DNT and time to EVT as well as lower reperfusion rates [13-15].

Although our AIS protocol [7, 8] has remained unchanged during the COVID-19 epidemic, the circumstances may change. All patients are triaged and screened for COVID-19. If the suspected AIS patient has symptoms associated with SARS-CoV-2, the patient is treated in a separate cohort area in our ED. Examination and treatment are performed with full personal protective equipment (PPE), likewise laboratory testing and radiology services in a CT room reserved for cohort patients. Nasal swab testing for SARS-CoV-2 is performed immediately after POC INR. Treatment of the patient requires 
TA B L E 1. Overview of the cases

\begin{tabular}{|c|c|c|c|c|c|c|c|}
\hline Patient & Time & Symptoms and signs & CT Findings & $\begin{array}{l}\text { Door-to- } \\
\text { CT } \\
\text { Time }\end{array}$ & $\begin{array}{c}\text { Door-to-CTA } \\
\text { Time }\end{array}$ & $\begin{array}{l}\text { Door-to- } \\
\text { Needle } \\
\text { Time }\end{array}$ & $\begin{array}{l}\text { Door-in- } \\
\text { Door-out } \\
\text { Time }\end{array}$ \\
\hline Man, 63 years & April 2020 & $\begin{array}{l}\text { Right-side hemiplegia, aphasia, } \\
\text { subtotal facial paresis, ataxia, } \\
\text { ocular deviation, lowered } \\
\text { consciousness }\end{array}$ & $\begin{array}{c}\text { Left } \\
\text { M1-thrombus }\end{array}$ & $5 \mathrm{~min}$. & $8 \mathrm{~min}$. & $11 \mathrm{~min}$. & $21 \mathrm{~min}$. \\
\hline Man, 81 years & May 2020 & $\begin{array}{l}\text { Right-side hemiplegia, aphasia, } \\
\text { ocular deviation, subtotal facial } \\
\text { paresis, lowered consciousness }\end{array}$ & $\begin{array}{c}\text { Left } \\
\text { M1-thrombus }\end{array}$ & $9 \mathrm{~min}$. & $\begin{array}{c}16 \text { min. } \\
\text { (After first } \\
\text { bolus of tPA) }\end{array}$ & $10 \mathrm{~min}$. & $19 \mathrm{~min}$. \\
\hline Man, 82 years & July 2020 & $\begin{array}{l}\text { Left-side hemiplegia, ocular } \\
\text { deviation, neglect, subtotal facial } \\
\text { paresis, lowered consciousness }\end{array}$ & $\begin{array}{c}\text { Right } \\
\text { M2-thrombus }\end{array}$ & $5 \mathrm{~min}$. & $7 \mathrm{~min}$. & N/A & $15 \mathrm{~min}$. \\
\hline
\end{tabular}

seamless co-operation and concentration of EMS and ED staff to minimise possible delays in thrombolytic and endovascular treatments.

Our ultra-fast diagnostic protocol applicable to AIS patients in PSC settings described above has also been active during COVID-19. Here we present experiences with the protocol using three case-studies of patients who received EVT.

\section{Case presentations}

Case 1, April 2020: The patient was a 63-year-old male. His medical history included arterial hypertension treated with candesartan $8 \mathrm{mg}$ once a day as the only regular medication.

On Monday at 19:10, the patient suffered sudden rightside hemiplegia. He was able to call his wife, who at 19:30 contacted the dispatch centre by dialling 112. After EMS arrived on the scene, the paramedics consulted an emergency physician and the stroke protocol [8] was initiated.

The patient arrived at our ED by an ambulance at 20:19. His primary National Institute of Health Stroke Scale (NIHSS) score in the ED was 20 points (subtotal facial nerve paralysis on the right, together with hemiplegic right arm and leg, ocular deviation to the left, severe aphasia and dysarthria). Blood pressure was $139 / 102 \mathrm{mmHg}$, pulse rate 110 beats per minute (bpm), saturation 96\%, and POC INR 1.1.

NCCT at 20:24 showed no contraindication for thrombolysis and the first bolus of alteplase was given immediately after the CTA at 20:30, i.e. eleven minutes after arrival at the ED. The radiologist on call reported orally that according to the CTA the patient had thrombus in the M1-segment of the left middle cerebral artery (M1-thrombus) (Table 1).

Paramedics were awaiting the results, and the patient left for thrombectomy in Tampere University Hospital at 20:40. DIDO time was thus 21 minutes (Table 1). There were no complications due to thrombolysis.

Case 2, May 2020: An 81-year old man had a cardiac VVI pacemaker due to atrioventricular block, mild aortic sclerosis, cardiac insufficiency, paroxysmal atrial fibrillation, arterial hypertension, hypercholesterolemia, type 2 diabetes mellitus and chronic obstructive pulmonary disease (COPD). He was an ex-smoker. Last mini-mental state exam score was 27/30. In spite of his medical history, the patient was in stable mental health and able to live independently. He had visited the cardiology outpatient clinic nine days earlier and had been diagnosed with paroxysmal atrial fibrillation. Direct oral anticoagulant (DOAC) medication was then prescribed.

On Monday at 13:15, the patient's wife had noticed that he had right-side hemiplegia. The paramedics arrived on the scene at 13:37 and found that the patient had right-side hemiplegia without ocular deviation. After consulting an emergency physician, they initiated the stroke protocol.

The patient was taken to the ED at 14:12. His primary NIHSS score in the ED was at least 19 points (lowered consciousness, total aphasia, ocular deviation to the left, subtotal facial nerve paralysis on the right side, together with hemiplegic right arm and leg). Due to his severe symptoms, he could not be tested reliably for neglect, ataxia or visual impairment. Blood pressure was $135 / 83 \mathrm{mmHg}$, pulse rate 91 bpm, saturation $94 \%$ without oxygen support, blood glucose $8.4 \mathrm{mmol} / \mathrm{L}$, and POC INR 1.2. According to the meticulous gathering of information from the electronic patient record, the patient's wife and the cardiologist who had prescribed the DOAC, we were able to conclude that the patient had not begun DOAC therapy.

NCCT (performed at 14:21) showed no contraindication for thrombolysis. The first bolus was given at 14:22, i.e. 10 minutes after arriving at the ED. tPA bolus was given and the CTA was performed at 14:28. According to the oral information of the radiologist, the CTA confirmed a left M1thrombus. At 14:31 with DIDO time of 19 minutes, the patient left with paramedics for thrombectomy in Tampere University Hospital (Table 1). There were no complications due to thrombolysis.

Case 3, July 2020: The patient was an 82-year-old man. According to his medical history, the patient had COPD, operated aortic valve (bio valve) and chronic atrial fibrillation. He was in good physical health and able to live independently.

On Thursday at 12:00, the patient suffered a sudden leftside hemiplegia with ocular deviation to the right. Paramedics arrived on the scene and confirmed the primary findings. After consulting an emergency physician, they initiated the stroke protocol.

The patient arrived at the ED by ambulance at 13:09. His primary NIHSS score in the ED was 24 points (lowered con- 
sciousness, unable to follow instructions, ocular deviation to the right, left subtotal facial nerve paralysis, both left-side extremities were hemiplegic, severe aphasia, severe dysarthria and neglect). Blood pressure was $184 / 81 \mathrm{mmHg}$, pulse rate 91 bpm, saturation $92 \%$ without oxygen supplement, and glucose $7.7 \mathrm{mmol} / \mathrm{L}$.

NCCT performed at 13:14 showed no contraindications for thrombectomy. Due to DOAC usage, thrombolysis was contraindicated. According to the CTA (oral information from the radiologist), the patient had right-side M2-thrombus. Paramedics left the ED at 13:24 to transport the patient for thrombectomy at Tampere University Hospital (Table 1).

\section{Discussion}

The three patients presented here were selected as examples of the functionality of our protocol during COVID-19 epidemic. Diagnostic practice for these patients with AIS and need for EVT were very straightforward without unnecessary delays. This was possible because of our lean AIS process, where paramedics, emergency physicians, nurses, laboratory technicians and the staff of the radiology unit have organized seamless co-operation $[7,8]$.

One big provider operates the prehospital EMS of our hospital district with medical leadership run by our ED. This enables equal training and practices for all paramedics. Every paramedic is therefore aware of and able to follow our precise and straightforward stroke protocol.

In our hospital district, primary and secondary heath care in the public sector have shared electronic health records (EHR). Data from private health care providers with their own EHR are available through the National eHealth Archive [16]. The joint organization, pre-notification from the EMS and the new e-technology with shared health data enable emergency physicians to familiarize themselves with the history of the stroke patient prior to arrival at the ED. Further, the single call activation system enables ED and CT staff to prepare for the AIS patient's reception before the patient arrives at the ED [7, 8]. Together with the medical history and clinical examination we should distinguish between stroke mimics and AIS. The process described here with very short DIDOs is in accordance with the strong recommendations of earlier studies when aiming at better outcomes for AIS patients with LVOs [17-19].

Despite the ongoing COVID-19 epidemic, the patients in this study were treated like any other AIS patients according to our stroke protocol. None of them had symptoms indicative of COVID-19. Patients with SARS-CoV-2 may be at increased risk of thrombosis [20-22]. We treat patients with infections, including COVID-19, in a separate cohort area of our ED. Whether the time intervals of AIS patients with infections are comparable to those without is not clear. With other SARS$\mathrm{CoV}-2$ suspected or confirmed patients the treatment times are longer. We suspect the reasons behind this are low resources and limited preparedness. Personnel, space, equipment and supplies are limited. Therefore, the patient flow decreases and the treatment times are increasing.

In patients with emergent LVO, long DIDO time seems to have a deleterious effect on outcome and may be the most important modifiable factor in onset to recanalization time in a PSC [14, 23]. Although longer median times have been reported [17, 19, 24], it has recently been shown that it is possible to achieve a median DIDO time of 60 minutes [19]. In this case report on three AIS patients with very short time intervals, we did not study the median DIDO time, which may constitute a weakness. We are continuously analysing the processes of our ED [25-28] including the time intervals of the AIS patients in order to publish not only median DNTs $[7,8]$ but also eventually median DIDO times. According to our data during the COVID-19 epidemic, there have been 14 patients with DIDO times. Of these, $43 \%$ had shorter DIDO time of less than 25 minutes.

In conclusion, it is of great importance to diagnose all patients with acute ischaemic stroke immediately in the emergency department. In a primary stroke centre, patients needing endovascular treatment should achieve short door-in-door-out time. Following this principle, our report on three patients exemplifies efficient co-operation between EMS and the ED together with a smooth diagnostic process for AIS patients in the ED.

\section{ABBREVIATIONS}

AIS, Acute Ischaemic Stroke; COPD, Chronic Obstructive Pulmonary Disease; COVID-19, Corona Virus Disease-19; CT, Computed Tomography; CTA, Computed Tomography Angiography; DIDO, Door-in-Door-out; DNT, Door-toNeedle Time; DOAC, Direct Oral Anticoagulant; ED, Emergency Department; EHR, Electronic Health Record; EMS, Emergency Medical Service; EVT, Endovascular Treatment; LVO, Large Vessel Occlusion; M1-thrombus, M1-segment (sphenoidal or horizontal segment) of the Middle Cerebral Artery; M2-thrombus, M2-segment (insular segment) of the Middle Cerebral Artery; NCCT, Baseline Non-Contrast Computed Tomography; NIHSS, National Institutes of Health Stroke Scale; POC, Point-of-Care; PPE, Personal Protective Equipment; PSC, Primary Stroke Centre; SARS-CoV-2, Severe acute respiratory syndrome coronavirus 2; tPA, tissue Plasminogen Activator.

\section{AUTHOR CONTRIBUTIONS}

MG and AP planned the study, TT, MG and VH gathered the material. $\mathrm{MG}, \mathrm{VH}, \mathrm{TT}$ and AP drafted the manuscript. All authors read and approved the final manuscript.

\section{ETHICS APPROVAL AND CONSENT TO PARTICIPATE}

We requested approval from the Ethics Committee of Pirkanmaa Hospital District. However, this was waived because according to the Finnish legislation, register examinations in which the subjects are not in contact/are not subject to intervention do not require the permission from the ethics committee. 


\section{ACKNOWLEDGMENT}

The authors gratefully acknowledge the professional linguistic aid of Mrs. Virginia Mattila as well as the co-operation of the personnel of the EMS and ED in Kanta-Häme Hospital District.

\section{FUNDING}

This study was supported by grants from the Ministry of Health and Social Welfare in Finland through the Medical Research Fund of Kanta-Häme Central Hospital and Pirkanmaa Hospital District.

\section{CONFLICT OF INTEREST}

The authors have no competing interests.

\section{REFERENCES}

[1] Jauch EC, Saver JL, Adams HP Jr, Bruno A, Connors JJ, Demaerschalk $\mathrm{BM}$, et al. Guidelines for the early management of patients with acute ischemic stroke: a guideline for healthcare professionals from the American Heart Association/American Stroke Association. Stroke. 2013; 44: 870-947.

[2] Emberson J, Lees KR, Lyden P, Blackwell L, Albers G, Bluhmki E, et al. Effect of treatment delay, age, and stroke severity on the effects of intravenous thrombolysis with alteplase for acute ischaemic stroke: a meta-analysis of individual patient data from randomised trials. The Lancet. 2014; 384: 1929-1935.

[3] Sarraj A, Sangha N, Hussain MS, Wisco D, Vora N, Elijovich L, et al. Endovascular therapy for acute ischemic stroke with occlusion of the middle cerebral artery M2 segment. JAMA Neurology. 2016; 73: 12911296.

[4] Román LS, Menon BK, Blasco J, Hernández-Pérez M, Dávalos A, Majoie CBLM, et al. Imaging features and safety and efficacy of endovascular stroke treatment: a meta-analysis of individual patient-level data. The Lancet Neurology. 2018; 17: 895-904.

[5] Saver JL, Goyal M, van der Lugt A, Menon BK, Majoie CB, Dippel DW, et al. Time to treatment with endovascular thrombectomy and outcomes from ischemic stroke: a meta-analysis. Journal of the American Medical Association. 2016; 316: 1279-1288.

[6] Naskali J, Palomaki A, Harjola VP, Hallberg V, Rautava VP, Innamaa T. Emergency medicine in Finland: first year experiences of specialist training. Journal of Academic Emergency Medicine. 2014; 13: 26-29.

[7] Heikkilä I, Kuusisto H, Stolberg A, Palomäki A. Stroke thrombolysis given by emergency physicians cuts in-hospital delays significantly immediately after implementing a new treatment protocol. Scandinavian Journal of Trauma, Resuscitation and Emergency Medicine. 2016; 24: 46.

[8] Heikkilä I, Kuusisto H, Holmberg M, Palomäki A. Fast protocol for treating acute ischemic stroke by emergency physicians. Annals of Emergency Medicine. 2019; 73: 105-112.

[9] Distante C, Piscitelli P, Miani A. COVID-19 outbreak progression in Italian regions: approaching the peak by the end of March in northern Italy and first week of April in southern Italy. International Journal of Environmental Research and Public Health. 2020; 17: 3025.

[10] Alquézar-Arbé A, Piñera P, Jacob J, Martín A, Jiménez S, Llorens P, et al. Impact of the COVID-19 pandemic on hospital emergency departments: results of a survey of departments in 2020-the Spanish ENCOVUR study. Emergencias. 2020; 32: 320-331.

[11] Mao L, Jin H, Wang M, Hu Y, Chen S, He Q, et al. Neurologic manifestations of hospitalized patients with coronavirus disease 2019 in Wuhan, China. JAMA Neurology. 2020; 77: 683.

[12] Ellul MA, Benjamin L, Singh B, Lant S, Michael BD, Easton A, et al.
Neurological associations of COVID-19. The Lancet Neurology. 2020; 19: 767-783.

[13] Tejada Meza H, Lambea Gil Á, Sancho Saldaña A, Martínez-Zabaleta M, Garmendia Lopetegui E, López-Cancio Martínez E, et al. Impact of COVID-19 outbreak in reperfusion therapies of acute ischaemic stroke in northwest Spain. European Journal of Neurology. 2020; 27: 2491-2498.

[14] Plumereau C, Cho TH, Buisson M, Amaz C, Cappucci M, Derex L, et al. Effect of the COVID-19 pandemic on acute stroke reperfusion therapy: data from the Lyon Stroke Center Network. Journal of Neurology. 2020. doi: 10.1007/s00415-020-10199-6 (online ahead of print).

[15] Bhaskar S, Sharma D, Walker AH, McDonald M, Huasen B, Haridas A, et al. Acute neurological care in the COVID-19 era: the pandemic health system REsilience PROGRAM (REPROGRAM) consortium pathway. Frontiers in Neurology. 2020; 11: 579.

[16] Ruotsalainen P, Iivari A, Doupi P. Finland's strategy and implementation of citizens' access to health information. Studies in Health Technology and Informatics. 2008; 137: 379-385.

[17] McTaggart RA, Moldovan K, Oliver LA, Dibiasio EL, Baird GL, Hemendinger ML, et al. Door-in-door-out time at primary stroke centers may predict outcome for emergent large vessel occlusion patients. Stroke. 2018; 49: 2969-2974.

[18] Choi PMC, Tsoi AH, Pope AL, Leung S, Frost T, Loh P, et al. Doorin-door-out time of 60 minutes for stroke with emergent large vessel occlusion at a primary stroke center. Stroke. 2019; 50: 2829-2834.

[19] Tiu J, Watson T, Clissold B. Mechanical thrombectomy for emergent large vessel occlusion: an Australian primary stroke centre workflow analysis. Internal Medicine Journal. 2020. doi: 101111/imj.14843 (online ahead of print).

[20] Panigada M, Bottino N, Tagliabue P, Grasselli G, Novembrino C, Chantarangkul V, et al. Hypercoagulability of COVID-19 patients in intensive care unit: a report of thromboelastography findings and other parameters of hemostasis. Journal of Thrombosis and Haemostasis. 2020; 18: 1738-1742.

[21] Ranucci M, Ballotta A, Di Dedda U, Bayshnikova E, Dei Poli M, Resta $\mathrm{M}$, et al. The procoagulant pattern of patients with COVID-19 acute respiratory distress syndrome. Journal of Thrombosis and Haemostasis. 2020; 18: 1747-1751.

[22] Maier CL, Truong AD, Auld SC, Polly DM, Tanksley C, Duncan A. COVID-19-associated hyperviscosity: a link between inflammation and thrombophilia? The Lancet. 2020; 395: 1758-1759.

[23] Santana Baskar P, Cordato D, Wardman D, Bhaskar S. In-hospital acute stroke workflow in acute stroke-systems-based approaches. Acta Neurologica Scandinavica. 2021; 143: 111-120.

[24] Gangadharan S, Lillicrap T, Miteff F, Garcia-Bermejo P, Wellings T, O'Brien B, et al. Air vs. Road decision for endovascular clot retrieval in a rural telestroke network. Frontiers in Neurology. 2020; 11: 628.

[25] Rautava V, Palomäki E, Innamaa T, Perttu M, Lehto P, Palomäki A. Improvement in self-reported confidence in nurses' professional skills in the emergency department. Scandinavian Journal of Trauma, Resuscitation and Emergency Medicine. 2013; 21: 16.

[26] Lehtonen H, Lukkarinen T, Kämäräinen V, Rautava VP, Parviainen P, Palomäki A. Improving emergency department capacity efficiency. Signa Vitae. 2016; 12: 52-57.

[27] Ojakäär A, Purdy M, Kechagias A, Järvelin U, Palomäki A. Immediate effects of urgent reorganisation of emergency department-based treatment pathway in nonperforated appendicitis: a retrospective study. BMC Emergency Medicine. 2020; 20: 45.

[28] Tuominen J, Hällberg V, Oksala N, Palomäki A, Lukkarinen T, Roine A. NYU-EDA in modelling the effect of COVID-19 on patient volumes in a Finnish emergency department. BMC Emergency Medicine. 2020; 20: 97.

How to cite this article: Markku Grönroos, Ville Hällberg, Tuukka Tomminen, Ari Palomäki. Short door-in-door-out time of stroke patients in need of thrombectomy in a primary stroke centre. Signa Vitae. 2021;17(3):242-245. doi:10.22514/sv.2021.039. 